\title{
3-Connected planar graphs are 5-distinguishing colorable with two exceptions*
}

\author{
Gašper Fijavž \\ Faculty of Computer and Information Science, University of Ljubljana \\ Tržaška 25, 1000 Ljubljana, Slovenia. \\ Seiya Negami \\ Faculty of Education and Human Sciences, Yokohama National University \\ 79-2 Tokiwadai, Hodogaya-Ku, Yokohama 240-8501, Japan. \\ Terukazu Sano \\ Graduate School of Environment and Information Sciences, Yokohama National \\ University, 79-7 Tokiwadai, Hodogaya-Ku, Yokohama 240-8501, Japan.
}

Received 31 March 2010, accepted 23 January 2011, published online 19 March 2011

\begin{abstract}
A graph $G$ is said to be $d$-distinguishing colorable if there is a $d$-coloring of $G$ such that no automorphism of $G$ except the identity map preserves colors. We shall prove that every 3 -connected planar graph is 5-distinguishing colorable except $K_{2,2,2}$ and $C_{6}+\bar{K}_{2}$ and that every 3 -connected bipartite planar graph is 3-distinguishing colorable except $Q_{3}$ and $R\left(Q_{3}\right)$.
\end{abstract}

Keywords: planar graphs, distinguishing number, distinguishing chromatic number.

Math. Subj. Class.: 05C15, 05C10

\section{Introduction}

A coloring or a $k$-coloring of a graph $G$ is an assignment of $k$ colors to its vertices so that every pair of adjacent vertices gets different colors and the chromatic number $\chi(G)$ of $G$ is defined as the minimum number $k$ such that $G$ admits a $k$-coloring, as usual. Even if a graph itself has rich symmetry, its coloring does not adapt to symmetry in general

\footnotetext{
* Dedicated to the memory of Michael Albertson.

E-mail addresses: gasper.fijavz@ fri.uni-lj.si (Gašper Fijavž), negami@edhs.ynu.ac.jp (Seiya Negami), d08tc004@ynu.ac.jp (Terukazu Sano)
} 
and it might become completely asymmetric. How many colors do we need to break the symmetry of a graph? We formulate this consideration as follows.

Let $c: V(G) \rightarrow\{1, \ldots, k\}$ be a coloring of a graph $G$. We denote by $\operatorname{Aut}(G, c)$ the set of automorphisms $\sigma$ over $G$ that preserve the colors given by $c$.

$$
\operatorname{Aut}(G, c)=\{\sigma \in \operatorname{Aut}(G): c(\sigma(v))=c(v) \text { for all } v \in V(G)\}
$$

It is clear that $\operatorname{Aut}(G, c)$ forms a subgroup in the automorphism group $\operatorname{Aut}(G)$ of $G$. If $\operatorname{Aut}(G, c)$ is trivial, that is, if it includes only the identity map $\operatorname{id}_{G}$, then $c$ is said to be distinguishing or is called a $k$-distinguishing coloring involving the number of colors $k$. If $G$ admits a $k$-distinguishing coloring, then $G$ is said to be $k$-distinguishing colorable. The distinguishing chromatic number of $G$ is defined as the minimum number $k$ such that $G$ is $k$-distinguishing colorable and is denoted by $\chi_{D}(G)$.

A planar graph is one that can be drawn in the plane without crossings on edges. It is well-known that every planar graph is 4-colorable, by the "Four Color Theorem", that is, the chromatic number of planar graphs is bounded by 4 . We would like to establish a similar theorem for the distinguishing chromatic number of planar graphs. However, there is no finite upper bound for it; it is easy to see that $\chi_{D}\left(K_{2, n}\right)=n+2$ for example. Thus, we need some assumption on graphs to bound the distinguishing chromatic number in general.

A graph is said to be $n$-connected if it has at least $n+1$ vertices and if removing fewer than $n$ vertices does not disconnect it. Recently, Negami and Sakurai [6] have proved the following theorem on planar graphs as an application of general arguments on the distinguishing chromatic number of graphs faithfully embedded on closed surfaces; we shall explain later the faithfulness of embedding.

Theorem 1.1 (Negami and Sakurai [6]). Every 3-connected planar graph is 6-distinguishing colorable.

This theorem is best possible in two meanings. First, the assumption of being 3connected cannot be omitted. For example, $K_{2, n}$ is not 3-connected and its distinguishing chromatic number can be arbitrarily large, as mentioned above. Secondly, there exist 3-connected planar graphs that are not 5-distinguishing colorable, as follows.

Let $C_{n}+\bar{K}_{2}$ denote the suspension over a cycle $C_{n}$ of length $n$, that is, $C_{n}$ with two extra vertices adjacent to all vertices along $C_{n}$. This may be called a double wheel with rim $C_{n}$. In particular, $C_{4}+\bar{K}_{2}$ is isomorphic to $K_{2,2,2}$, which is the octahedron. The double wheels are 5-distinguishing colorable except two; $\chi_{D}\left(K_{2,2,2}\right)=\chi_{D}\left(C_{6}+\bar{K}_{2}\right)=6$. They are 3 -connected and can be embedded on the sphere as a triangulation, that is, so that each face is triangular. A graph having such an embedding is said to be maximal planar in general and adding any new edge to it results in a nonplanar graph.

Negami and Sakurai [6] have proved that every maximal planar graph is 5-distinguishing colorable if it is isomorphic to neither of these two exceptions and conjectured that every 3-connected planar graph is 5-distinguishing colorable with a finite number of exceptions. In this paper, we shall solve their conjecture affirmatively and show that the exceptions are the two listed above:

Theorem 1.2. Every 3-connected planar graph is 5-distinguishing colorable unless it is isomorphic to either $K_{2,2,2}$ or $C_{6}+\bar{K}_{2}$. 
It has been shown in [6] that $\chi_{D}\left(C_{n}+\bar{K}_{2}\right)=5$ if $n \neq 4,6$ and hence we cannot improve the above theorem even if we allow a finite number of exceptions. However, there are many planar graphs whose distinguishing chromatic number is less than 5 . For example, it is not so difficult to show that every 3 -connected bipartite planar graph is 4-distinguishing colorable. We shall prove a stronger fact on bipartite planar graphs. In general, the radial graph $R(G)$ of a graph $G$ embedded on the sphere is defined as the graph obtained as follows; put a vertex in each face of $G$, join it to all vertices lying along the boundary cycle of the face, and finally delete all original edges of $G$ :

Theorem 1.3. Every 3-connected bipartite planar graph is 3-distinguishing colorable unless it is isomorphic to either the 3-cube $Q_{3}$ or its radial graph $R\left(Q_{3}\right)$.

The distinguishing chromatic number of graphs has been introduced in [3], as a variant of the distinguishing number. The latter can be defined in the same way as for the former without the assumption of $c$ being a proper coloring in the previous; $c$ is just an assignment of numbers to vertices and adjacent vertices may have the same number. There have been a lot of studies about the distinguishing number of graphs, see [1, 2, 4].

\section{General case}

The point of our arguments below is the fact that a 3-connected planar graph can be embedded on the sphere so that any automorphism $\sigma$ of $G$ extends to an auto-homeomorphism $h$ over the sphere with $\left.h\right|_{G}=\sigma$. Such an embedding is said to be faithful. The notion of faithful embeddings has been introduced in [5] and the faithfulness of an embedding of any 3-connected planar graph on the sphere can be derived from the uniqueness of its dual, proved by Whitney [8].

To conclude that a given graph is $k$-distinguishing colorable, we must show that every automorphism $\sigma \in \operatorname{Aut}(G, c)$ is the identity map for a suitable $k$-coloring of $G$. The following lemma has been proved and used to do it in $[4,6]$ and also we shall often use it implicitly.

Lemma 2.1. Let $G$ be a 3-connected planar graph embedded on the sphere and $\sigma$ its automorphism. If $\sigma$ fixes all vertices lying along the boundary cycle of a face, then $\sigma$ is the identity map.

As is mentioned in the Introduction, Theorem 1.2 has been proved in the case of maximal planar graphs in [6]. However, we shall include our own arguments for such a case in the proof below to make it self-contained. An $n$-cut in a connected graph is a set of $n$ vertices whose removal results in a disconnected graph. The following lemma is useful to discuss the distinguishing chromatic number of triangulations:

Lemma 2.2. If a triangulation $G$ on the sphere has a 3-cut, then $\chi_{D}(G) \leq \chi(G)+1$.

Proof. In general, the three vertices of any 3 -cut in a triangulation $G$ form a cycle of length 3 and it separates the vertices of $G$ into two groups. Call such a cycle a separating 3cycle here. If $G$ is embedded on the plane, we can distinguish the inside and outside of a separating 3-cycle and can consider an innermost separating 3-cycle, that is, one such that its inside contains no other separating 3 -cycles.

Let $C=v_{1} v_{2} v_{3}$ be an innermost separating 3 -cycle of $G$ and choose a vertex $x$ inside $C$. Consider a $k$-coloring $\bar{c}: V(G) \rightarrow\{1, \ldots, k\}$ of $G$ with $\bar{c}\left(v_{i}\right)=i$ for $i=1,2,3$ and 
change the color of $x$ to $k+1$ to obtain a $(k+1)$-coloring $c$ of $G$. Clearly, any automorphism $\sigma \in \operatorname{Aut}(G, c)$ fixes $x$ and cannot move $C$ into its inside since it is innermost. This implies that $\sigma(C)=C$ and hence $\sigma$ fixes each of $v_{1}, v_{2}$ and $v_{3}$ since they have different colors. Consider another triangulation $G^{\prime}$ obtained from $G$ by deleting all vertices lying in the inside of $C$. Then the region inside $C$ is a face of $G^{\prime}$ with boundary cycle $C$ and $\sigma$ induces naturally an automorphism $\sigma^{\prime}$ of $G^{\prime}$ such that it fixes the face. Applying Lemma 2.1 to $\sigma^{\prime}$, we conclude that $\sigma^{\prime}$ is the identity map of $G^{\prime}$ and hence $\sigma$ is the identity map of $G$ since $\sigma$ fixes the faces outside $C$. Therefore, $G$ is $(k+1)$-distinguishing colorable.

The neighbors of a vertex in a triangulation $G$ on a closed surface form a cycle and such a cycle is called its link. If a triangulation $G$ on the sphere has a vertex of degree 3 , then the link of the vertex becomes a separating 3-cycle of $G$ and $G$ is not 4-connected. If a triangulation $G$ on the sphere is not 4-connected in general, then we have $\chi_{D}(G) \leq$ $\chi(G)+1$ by Lemma 2.2 and hence $G$ is 5 -distinguishing colorable since $\chi(G) \leq 4$ by Four Color Theorem.

Let $G$ be a 3-connected graph embedded on the plane or on the sphere. Then each face of $G$ is bounded by a cycle. The size of a face $A$ in $G$ is defined as the length of the boundary cycle of $A$ and is denoted by $|A|$. We call a face $A$ an even face or an odd face below, according to the parity of $|A|$.

Here we shall prove Theorem 1.2, that is, show that every 3-connected planar graph is 5-distinguishing colorable, specifying the two exceptions.

Proof of Theorem 1.2. Let $G$ be a 3-connected planar graph and suppose that it is faithfully embedded on the sphere. By Four Color Theorem, $G$ has a coloring $\bar{c}$ with at most four colors. We shall modify it to a 5 -distinguishing coloring $c$ in each of the following cases.

CASE 1. There is no odd face: In this case, $G$ is a bipartite planar graph and hence it has a 2-coloring $\bar{c}: V(G) \rightarrow\{1,2\}$. We can find four distinct vertices $x_{1}, y_{1}, x_{2}$ and $y_{2}$ so that $x_{1} y_{1} x_{2}$ and $y_{1} x_{2} y_{2}$ form corners of two faces sharing an edge $y_{1} x_{2}$, that is, they are paths of length 2 lying along the boundary cycles of the faces. Consider a 4-coloring $c$ of $G$ defined by $c\left(x_{1}\right)=c\left(x_{2}\right)=3, c\left(y_{1}\right)=c\left(y_{2}\right)=4$ and $c(v)=\bar{c}(v)$ for the other vertices $v$. It is clear that any automorphism $\sigma \in \operatorname{Aut}(G, c)$ fixes the two corners $x_{1} y_{1} x_{2}$ and $y_{1} x_{2} y_{2}$ and $\sigma$ is the identity map. Therefore, $c$ is a 4 -distinguishing coloring and hence $\chi_{D}(G) \leq 4$ in this case.

CASE 2. There is an odd face of size at least 5: Consider a 4-coloring $\bar{c}: V(G) \rightarrow$ $\{1,2,3,4\}$ of $G$. Let $A$ be an odd face of $G$ with $|A|=2 m+1 \geq 5$ and $C=v_{0} v_{1} \cdots v_{2 m}$ its boundary cycle. Define a 5 -coloring $c$ of $G$ by $c\left(v_{1}\right)=c\left(v_{2 m}\right)=5$ and $c(w)=\bar{c}(w)$ for the other vertices $w$. Take any automorphism $\sigma \in \operatorname{Aut}(G, c)$ with its extension $h$ over the sphere. If $h(A) \neq A$, then the two faces $h(A)$ and $A$ meet each other in $\left\{v_{1}, v_{2 m}\right\}$, which would form a 2 -cut of $G$, contrary to $G$ being 3 -connected. Therefore, we conclude that $h(A)=A$ and hence $\sigma(C)=C$.

It is clear that there are two possibilities on $\sigma$; it acts on $C$ as either the identity map or the reflexion exchanging $v_{1}$ and $v_{2 m}$. If the latter happened, then $\sigma$ would exchange $v_{m}$ and $v_{m+1}$, but this is impossible since they have different colors. Therefore, $\sigma$ is the identity map and hence $c$ is a 5-distinguishing coloring of $G$.

CASE 3. There exist odd faces and even faces, and all odd faces are of size 3: In this case, we can find two faces $A$ and $B$ with $|A|=3$ and $|B| \geq 4$ so that they share an edge $x y$. 
Consider the graph $G / x y$ obtained from $G$ by contracting $x y$. Since $G / x y$ might contain multiple edges but no self-loop, we can consider a coloring of $G / x y$. Since $G / x y$ is planar, $G / x y$ admits a 4-coloring and it induces a color assignment $c^{\prime}$ of $G$ with $c^{\prime}(x)=c^{\prime}(y)=4$; this $c^{\prime}$ is not a proper coloring of $G$. Define a 5-coloring $c: V(G) \rightarrow\{1,2,3,4,5\}$ of $G$ by $c(y)=5$ and $c(w)=c^{\prime}(w)$ for the other vertices $w$. Since the neighbors of $y$ except $x$ do not get color 4 in $c$, the edge $x y$ is a unique one which joins colors 4 and 5 . Thus, any automorphism $\sigma \in \operatorname{Aut}(G, c)$ fixes $x$ and $y$. Furthermore, $\sigma$ fixes $A$ and $B$ since $|A| \neq|B|$ and hence it is the identity map by Lemma 2.1. This implies that $c$ is a 5 -distinguishing coloring of $G$.

CASE 4. All faces are of size 3: If the three previous cases do not happen, then there is no even face and all faces are triangular. Thus, this case is the final one that we have to discuss. Now $G$ is a maximal planar graph embedded on the sphere as a triangulation. We may assume that $G$ is 4 -connected by Lemma 2.2.

First suppose that $G$ has a vertex $v$ of degree $d=5$ or $\geq 7$ and let $C=v_{0} v_{1} \cdots v_{d-1}$ be the link around $v$. If there exists an edge $v_{i} v_{i+3}$ in $G$, with indices taken modulo $d$, then $\left\{v, v_{i}, v_{i+3}\right\}$ would form a 3 -cut, contrary to $G$ being 4 -connected. Thus we may assume that there is no such edge in $G$. Consider a 4-coloring $\bar{c}$ of $G$. Then we can define a 5-coloring $c_{i}: V(G) \rightarrow\{1,2,3,4,5\}$ of $G$ for each $i \in\{0,1, \ldots, d-1\}$ by $c_{i}\left(v_{i}\right)=c_{i}\left(v_{i+3}\right)=5$ and $c_{i}(w)=\bar{c}(w)$ for the other vertices $w$ since there is no edge between $v_{i}$ and $v_{i+3}$.

If any automorphism $\sigma \in \operatorname{Aut}\left(G, c_{i}\right)$ fixes $v$, then it fixes $C$ and we conclude that $\sigma$ fixes each vertex lying along the link of $v$. For, the two vertices with color 5 cut $C$ into two segments of length 3 and $d-3(\neq 3)$. Since they have different lengths, $\sigma$ cannot swap them. Since $c\left(v_{i+1}\right) \neq c\left(v_{i+2}\right), \sigma$ cannot flip the segment $v_{i} v_{i+1} v_{i+2} v_{i+3}$, exchanging $v_{i}$ and $v_{i+3}$ and hence it fixes $C$. Thus, $c_{i}$ is a 5-distinguishing coloring of $G$ in this case. Otherwise, there is an automorphism $\sigma_{i} \in \operatorname{Aut}\left(G, c_{i}\right)$ with $\sigma_{i}(v) \neq v$ and $\sigma_{i}(v)$ is adjacent to both $v_{i}$ and $v_{i+3}$. If this happens for all $i$, then we find $d$ vertices $\sigma_{i}(v)$ outside the link of $v$ such that the cycle $v v_{i} \sigma_{i}(v) v_{i+3}$ separates $v_{i+1}$ and $v_{i+2}$ from the rest. The planarity forces all $\sigma_{i}(v)$ 's to be one vertex, and $G$ is isomorphic to $C_{d}+\bar{K}_{2}$. It is not difficult to see that $C_{d}+\bar{K}_{2}$ for $d=5$ or $\geq 7$ is 5 -distinguishing colorable and so is $G$.

Now we may suppose that every vertex in $G$ has degree at most 6 and not equal to 5. Since $G$ is 4 -connected, all vertices of $G$ have degree 4 or 6 and $G$ has a 3-coloring $\bar{c}: V(G) \rightarrow\{1,2,3\}$; in general, a maximal planar graph is 3 -colorable if and only if all vertices have even degree.

Let $v$ be one of vertices of degree 6 , if any, with link $C=v_{0} v_{1} v_{2} v_{3} v_{4} v_{5}$. Since $G$ is 4-connected, there is no edge between $v_{i}$ and $v_{i+3}$ for all $i$ and we can define a 5 -coloring $c_{i}: V(G) \rightarrow\{1,2,3,4,5\}$ of $G$ by $c_{i}\left(v_{i}\right)=c_{i}\left(v_{i+3}\right)=4, c_{i}\left(v_{i+1}\right)=5$ and $c(w)=\bar{c}(w)$ for the other vertices $w$. If any automorphism $\sigma \in \operatorname{Aut}\left(G, c_{i}\right)$ fixes $v$, then we conclude that $c_{i}$ is a 5-distinguishing coloring of $G$ by the asymmetric coloring along $C$. Otherwise, there is an automorphism $\sigma_{i} \in \operatorname{Aut}\left(G, c_{i}\right)$ such that $\sigma_{i}(v)$ lies outside $C$ and is adjacent to all of $v_{i}, v_{i+1}$ and $v_{i+3}$. If this happens for all $i$, then $G$ is isomorphic to $C_{6}+\bar{K}_{2}$ by the same logic as in the previous case.

On the other hand, if $G$ has no vertex of degree 6 , then all vertices have degree 4 and we can conclude that $G$ is isomorphic to $K_{2,2,2}$, which is one of the exceptions.

Finally we have shown that $G$ is 5 -distinguishing colorable or is isomorphic to one of the exceptions. Thus the theorem follows. 
Note 1: It is easy to show that a 3-connected planar graph $G$ is 6-distinguishing colorable, as follows. Suppose that $G$ is faithfully embedded on the sphere and let $u v w$ be a corner of a face $A$ of $G$. Contract the edge $u v$ to a vertex $[u v]$ and consider a 4-coloring $\bar{c}$ of the resulting graph $G / u v$ with $\bar{c}([u v])=4$. Using this, we can define a 6 -coloring $c$ of $G$ by $c(u)=4, c(v)=5, c(w)=6$ and $c(x)=\bar{c}(x)$ for any vertex $x$ other than $u, v$ and $w$. Then $u v$ is a unique edge with colors 4 and 5 at its ends and $A$ is a unique face having a corner with colors 4,5 and 6 . These force any automorphism $\sigma \in \operatorname{Aut}(G, c)$ to be the identity map. Therefore, $c$ is a 6 -distinguishing coloring.

This argument works to prove that $\chi_{D}(G) \leq \chi(G / u v)+2$ for a 3-connected planar graph $G$. On the other hand, Negami and Sakurai's proof of Theorem 1.1 concludes that $\chi_{D}(G) \leq \chi(G)+2$ unless $G$ is isomorphic to $K_{2,2,2}$ or $C_{6}+\bar{K}_{2}$. However, we cannot say which is better than the other. If $G$ is bipartite, then the latter implies that $\chi_{D}(G) \leq 4$, but the former does not; if $u v$ lies on a cycle, then we have $\chi(G / u v)=3$. If $G$ has an odd cycle and all odd cycles contain $u v$, then $G / u v$ is bipartite and the former implies that $\chi_{D}(G) \leq 4$, but the latter does not.

NOTE 2: We can construct infinitely many 3-connected planar graphs $G$ with $\chi_{D}(G)=5$, different from the series of $C_{n}+\bar{K}_{2}$, as follows. Let $G_{0}$ be the graph consisting of two triangles $u v w_{0}^{+}$and $u v w_{0}^{-}$sharing an edge $u v$ and another vertex $x$ of degree 4 adjacent to all of $u, v, w_{0}^{+}$and $w_{0}^{-}$. Then there is an automorphism $\sigma_{0}$ of $G_{0}$ which fixes $u, v$ and $x$, exchanging $w_{0}^{+}$and $w_{0}^{-}$. It is easy to see that $G_{0}$ is 4 -chromatic and has a unique 4-coloring, up to renaming colors. Add two extra vertices $w_{1}^{+}$and $w_{1}^{-}$to $G_{0}$ so that $w_{1}^{+}$ (respectively $w_{1}^{-}$) is adjacent to $u, v, w_{0}^{+}$(respectively $w_{0}^{-}$) in the resulting graph $G_{1}$. Then $\sigma_{0}$ extends naturally to be an automorphism $\sigma_{1}$ of $G_{1}$ with $\sigma_{1}\left(w_{1}^{ \pm}\right)=w_{1}^{\mp}$ and $G_{1}$ is uniquely 4-colorable as well as $G_{0}$.

Similarly, we can construct a series of 3-connected planar graphs $G_{0}, G_{1}, \ldots$, adding vertices $w_{i}^{+}$and $w_{i}^{-}$to $G_{i-1}$ so that each of $w_{i}^{ \pm}$is adjacent to three vertices which form a triangle in $G_{i-1}$ and that $G_{i}$ has an automorphism $\sigma_{i}$ exchanging $w_{j}^{+}$and $w_{j}^{-}$for all $j \leq i$ and fixing $u, v$ and $x$. Then $G_{i}$ is uniquely 4-colorable for all $i$. As evidenced by the existence of $\sigma_{i}$, the unique 4-coloring of $G_{i}$ is not a distinguishing coloring. This implies that $G_{i}$ is not 4 -distinguishing colorable and hence $\chi_{D}(G) \geq 5$. Since $G_{i}$ is 5 distinguishing colorable by Theorem 1.2, we have $\chi_{D}(G)=5$.

\section{Bipartite case}

We have already discussed the distinguishing chromatic number of 3-connected bipartite planar graphs in Case 1 in our proof of Theorem 1.2 and concluded that they are 4distinguishing colorable. This is a best possible result if we do not allow any exception:

Lemma 3.1. $\chi_{D}\left(Q_{3}\right)=4$ and $\chi_{D}\left(R\left(Q_{3}\right)\right)=4$.

Proof. Embed $Q_{3}$ in the 3 -space as a unit cube with vertices having coordinates $(x, y, z)$ $(x, y, z \in\{0,1\})$ and let $v_{x y z}$ be the vertex placed at $(x, y, z)$. To show that $\chi_{D}\left(Q_{3}\right) \geq 4$, let $c: V\left(Q_{3}\right) \rightarrow\{1,2,3\}$ be any 3-coloring. Then we may assume that color 1 is used for at most two vertices since $Q_{3}$ has eight vertices. There are three different 3-colorings, up to symmetry; color 1 appears only at $v_{000}$, or one more at $v_{110}$ or at $v_{111}$. Coloring of the other vertices with 2 and 3 is uniquely determined and we find a non-trivial automorphism preserving colors in each case. Thus, there is no 3-distinguishing coloring of $Q_{3}$ and hence $\chi_{D}\left(Q_{3}\right) \geq 4$. 

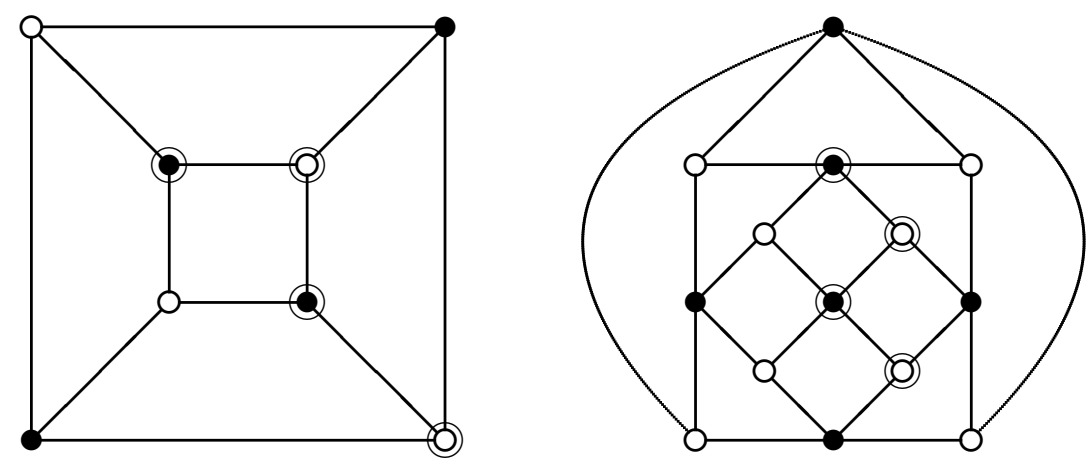

Figure 1: 4-Distinguishing colorings of $Q_{3}$ and $R\left(Q_{3}\right)$

Similarly, we can conclude that there is no 3-distinguishing coloring of $R\left(Q_{3}\right)$, as follows. The radial graph $R\left(Q_{3}\right)$ consists of the eight vertices belonging to $V\left(Q_{3}\right)$ and the six vertices corresponding to faces of $Q_{3}$. Suppose that $R\left(Q_{3}\right)$ has a 3-distinguishing coloring $c: V\left(R\left(Q_{3}\right)\right) \rightarrow\{1,2,3\}$ and let $\bar{c}$ be its restriction to the six vertices belonging to $V\left(R\left(Q_{3}\right)\right)-V\left(Q_{3}\right)$. If $\bar{c}$ uses only one color then the restriction of $c$ to $V\left(Q_{3}\right)$ induces a distinguishing coloring of $Q_{3}$. This implies that $c$ would use at least five colors for $R\left(Q_{3}\right)$ since $\chi_{D}\left(Q_{3}\right) \geq 4$. Thus, we may assume that $\bar{c}$ uses at least two colors. It should be noticed that three faces of $Q_{3}$ meeting at one vertex contain at most two colors; otherwise, the vertex would need the fourth color in $c$. Paying attention to this, we can find six candidates for $\bar{c}$, up to symmetry; one of them uses three colors and the other use two colors. For each coloring $\bar{c}$, we can find a plane in the 3 -space dividing the cube into halves and reflexing the coloring $c$. This is contrary to $c$ being a 3-distinguishing coloring. Therefore, we need at least four colors to construct a distinguishing coloring of $R\left(Q_{3}\right)$ and hence $\chi_{D}\left(R\left(Q_{3}\right)\right) \geq 4$.

By Case 1 in the proof of Theorem 1.2, we have known that a 3-connected bipartite planar graph has a 4-distinguishing coloring. For example, Figure 1 presents 4-distinguishing colorings of $Q_{3}$ and $R\left(Q_{3}\right)$. Therefore, we have $\chi_{D}\left(Q_{3}\right)=\chi_{D}\left(R\left(Q_{3}\right)\right)=4$.

Now we shall prove Theorem 1.3, that is, show that every 3 -connected bipartite graph is 3 -distinguishing colorable, except the two graphs given in the above lemma.

Proof of Theorem 1.3. Let $G$ be a 3-connected bipartite planar graph with partite sets $X$ and $Y$ and suppose that it is faithfully embedded on the sphere. We can define a 2-coloring $\bar{c}: V(G) \rightarrow\{1,2\}$ of $G$ with $\bar{c}(x)=1$ for $x \in X$ and $\bar{c}(y)=2$ for $y \in Y$ and any face of $G$ is bounded by a cycle of even length. We shall consider suitable 3-colorings $c, c^{\prime}, c_{i}: V(G) \rightarrow\{1,2,3\}$ many times, assigning color 3 to some specified vertices; the vertices other than those are always assumed to have the same colors as in $\bar{c}$.

CASE 1. $G$ is not a quadrangulation on the sphere: Since $G$ is 3-connected and bipartite, its dual $G^{*}$ is a simple planar graph with all vertices having even degree and there is no vertex of degree 2 in $G^{*}$. Since every planar graph has a vertex of degree at most $5, G^{*}$ has at least one vertex of degree 4 and the face of $G$ corresponding to this vertex of $G^{*}$ is quadrilateral. Thus, we can find a pair of faces $A$ and $B_{0}$ sharing one edge with $|A| \geq 6$ and $\left|B_{0}\right|=4$ since $G$ is not a quadrangulation. Let $C=v_{0} \cdots v_{d-1}$ be the boundary cycle 
of $A$ with $d \geq 6$ and $v_{0} v_{1} u_{0} w_{0}$ that of $B_{0}$. Define a 3-coloring $c: V(G) \rightarrow\{1,2,3\}$ of $G$ by $c\left(v_{0}\right)=c\left(v_{2}\right)=3$.

Take any automorphism $\sigma \in \operatorname{Aut}(G, c)$ and let $h$ be its extension over the sphere. Then we have $\sigma\left(\left\{v_{0}, v_{2}\right\}\right)=\left\{v_{0}, v_{2}\right\}$ since there is no vertex with color 3 other than $v_{0}$ and $v_{2}$. If $h(A) \neq A$, then the two faces $h(A)$ and $A$ meet each other in $\left\{v_{0}, v_{2}\right\}$, which would form a 2-cut of $G$, contrary to $G$ being 3 -connected. Therefore, we have $h(A)=A$ and $\sigma(C)=C$. It is clear that if $\sigma$ is not the identity map, then $\sigma$ acts on $C$ as a reflecion fixing $v_{1}$ and exchanging $v_{0}$ and $v_{2}$. This implies that there is a quadrilateral face $B_{1}$ with boundary cycle $\sigma\left(v_{1}\right) \sigma\left(v_{0}\right) \sigma\left(w_{0}\right) \sigma\left(u_{0}\right)=v_{1} v_{2} u_{1} w_{1}$. Repeat the same argument inductively as follows.

Suppose that we have found a quadrilateral face $B_{i}$ with boundary cycle $v_{i} v_{i+1} u_{i} w_{i}$. Then we redefine a 3-coloring $c$ by $c\left(v_{i}\right)=c\left(v_{i+2}\right)=3$. If $\operatorname{Aut}(G, c)$ contains only the identity map, then we can conclude that this $c$ is a 3-distinguishing coloring and hence $G$ is 3-distinguishing colorable. Otherwise, any automorphism $\sigma \in \operatorname{Aut}(G, c)$ acts on $C$ as a reflecion, fixing $v_{i+1}$ and exchanging $v_{i}$ and $v_{i+2}$, and we find another quadrilateral face $B_{i+1}$ with boundary cycle $\sigma\left(v_{i+1}\right) \sigma\left(v_{i}\right) \sigma\left(w_{i}\right) \sigma\left(u_{i}\right)=v_{i+1} v_{i+2} u_{i+1} w_{i+1}$. Therefore, if we have never concluded that $G$ is 3 -distinguishing colorable, we find a sequence of $d$ quadrilateral faces $B_{i}$ with boundary cycles $v_{i} v_{i+1} u_{i} w_{i}$ for $i \equiv 0, \ldots, d-1(\bmod d)$.

By the planarity, we find a non-adjacent pair $\left\{u_{i}, v_{i+3}\right\}$ for some $i$, say $i=0$, and can define a 3-coloring $c^{\prime}: V(G) \rightarrow\{1,2,3\}$ by $c^{\prime}\left(v_{0}\right)=c^{\prime}\left(u_{0}\right)=c^{\prime}\left(v_{3}\right)=3 ; v_{0}$ and $v_{3}$ are not adjacent since $\left\{v_{0}, v_{3}\right\}$ would form a 2 -cut of $G$ otherwise. Take any automorphism $\sigma \in \operatorname{Aut}\left(G, c^{\prime}\right)$ with its extension $h$ over the sphere. Since $v_{0}, u_{0} \in X$ and $v_{3} \in Y$, we have $\sigma\left(\left\{v_{0}, u_{0}\right\}\right)=\left\{v_{0}, u_{0}\right\}$ and $\sigma\left(v_{3}\right)=v_{3}$.

First suppose that $\sigma\left(v_{0}\right)=v_{0}$ for any automorphism $\sigma \in \operatorname{Aut}\left(G, c^{\prime}\right)$. Then $\sigma$ leaves $\left\{v_{0}, u_{0}\right\}$ and $\left\{v_{0}, v_{3}\right\}$ invariant, respectively. By the assumption of $G$ being 3 -connected, we have $h(A)=A$ and $h\left(B_{0}\right)=B_{0}$ and hence $\sigma$ fixes the edge $v_{0} v_{1}$ that $A$ and $B$ share. This implies that $h$ fixes $A$ and $B$ pointwise and hence $\sigma$ is the identity map. Therefore, $c^{\prime}$ is a 3 -distinguishing coloring in this case.

On the other hand, if $\sigma\left(v_{0}\right)=u_{0}$, then there is a face $h(A)(\neq A)$ of the same size as $A$ whose boundary cycle includes $u_{0}$ and $v_{3}$. This face blocks the adjacency between $u_{1}$ and $v_{4}$. In this case, we can redefine the 3-coloring $c^{\prime}$ so that $c^{\prime}\left(v_{1}\right)=c^{\prime}\left(u_{1}\right)=c^{\prime}\left(v_{4}\right)=3$ and the previous case will happen for $i=1$ since the face corresponding to $h(A)(\neq A)$ cannot exist. Therefore, $c^{\prime}$ is a 3 -distinguishing coloring, again.

CASE 2. $G$ is a quadrangulation on the sphere: We shall show that $G$ must be isomorphic to either $Q_{3}$ or $R\left(Q_{3}\right)$, assuming that $G$ is not 3-distinguishing colorable. Let $x$ be a vertex of degree $d \geq 5$ in $G$, if any, with neighbors $y_{0}, \ldots, y_{d-1}$ lying around it in this cyclic order and $x y_{i} x_{i} y_{i+1}$ the boundary cycles of $d$ faces $A_{i}$ incident to $x$ for $i \equiv 0, \ldots, d-1(\bmod d)$. We define a 3 -coloring $c_{i}: V(G) \rightarrow\{1,2,3\}$ by $c_{i}\left(y_{i}\right)=c_{i}\left(y_{i+1}\right)=c_{i}\left(x_{i+2}\right)=3$ if neither edge $y_{i} x_{i+2}$ nor $y_{i+1} x_{i+2}$ exists.

First suppose that there is an edge between $y_{0}$ and $x_{2}$. Then neither edge $y_{1} x_{3}$ nor $y_{2} x_{3}$ exists and we can define the 3 -coloring $c_{1}$. It is easy to see that an automorphism $\sigma \in \operatorname{Aut}\left(G, c_{1}\right)$ is the identity map if $\sigma$ fixes $x$. Thus, there is an automorphism $\sigma_{1} \in$ $\operatorname{Aut}\left(G, c_{1}\right)$ with $\sigma_{1}(x) \neq x$ since $G$ is assumed not to be 3 -distinguishing colorable. Let $h_{1}$ be the extension of $\sigma_{1}$ over the sphere. Since $\sigma_{1}\left(\left\{y_{1}, y_{2}\right\}\right)=\left\{y_{1}, y_{2}\right\}$, we have $\sigma_{1}(x)=x_{1}$ and there is a face $h_{1}\left(A_{3}\right)$ incident to both $x_{1}$ and $x_{3}$. However, the edge $y_{0} x_{2}$ blocks the existence of such a face. Thus, this is not the case and we may assume that there is no edge between $y_{i}$ and $x_{i+2}$ for all $i$. 
If there is an edge between $y_{1}$ and $x_{2}$, then neither edge $y_{d-1} x_{1}$ nor $y_{0} x_{1}$ exists and we can define the 3 -coloring $c_{d-1}$. Since $G$ is not 3 -distinguishing colorable, there is an automorphism $\sigma \in \operatorname{Aut}\left(G, c_{d-1}\right)$ which does not fix $x$, and we have $\sigma\left(\left\{y_{d-1}, y_{0}\right\}\right)=$ $\left\{y_{d-1}, y_{0}\right\}$. Then the extension $h$ of $\sigma$ over the sphere maps the face $A_{1}$ to a face incident to both $x_{d-1}$ and $x_{1}$. However, such a face does not exist by the planarity, a contradiction.

Therefore, we may assume that there is no edge between $y_{i+1}$ and $x_{i+2}$ in addition and hence we can define the 3 -coloring $c_{i}$ for all $i$. Furthermore, there is an automorphism $\sigma_{i} \in \operatorname{Aut}\left(G, c_{i}\right)$ with $\sigma_{i}(x)=x_{i}$; if $\sigma_{i}(x)=x$, then $\sigma_{i}$ is the identity map. Let $h_{i}$ be the extension of $\sigma_{i}$ over the sphere. Then $h_{0}\left(A_{0}\right)$ is a face incident to $x_{0}$ and $x_{2}$ and $h_{1}\left(A_{1}\right)$ is a face incident to $x_{1}$ and $x_{3}$. However, these faces block the existence of each other. Therefore, there exists no vertex of degree at least 5 in $G$.

Now we may assume that $G$ consists of only vertices of degree 3 and 4 since $G$ is 3-connected. Furthermore, we have $2|E(G)|=4|V(G)|-8$ by Euler's formula for a quadrangulation $G$ on the sphere. This implies that $G$ has exactly eight vertices of degree 3 in this case. To determine the structure of $G$, we shall show the following claim:

Claim 1. Let $x_{1} y_{1} x_{2} y_{2}$ and $x_{2} y_{2} x_{3} y_{3}$ be the boundary cycles of two faces $A_{1}$ and $A_{2}$ sharing an edge $x_{2} y_{2}$. Then $\operatorname{deg} y_{1}=\operatorname{deg} y_{3}$ and $\operatorname{deg} x_{1}=\operatorname{deg} x_{3}$.

First assume that $\operatorname{deg} y_{2}=4$ and let $y_{1}^{\prime}, x_{2}^{\prime}$ and $y_{3}^{\prime}$ be the three other vertices lying around $y_{3}$ so that $x_{1} y_{1}^{\prime} x_{2}^{\prime} y_{2}$ and $y_{2} x_{2}^{\prime} y_{3}^{\prime} x_{3}$ bound two faces incident to $y_{2}$ other than $A_{1}$ and $A_{2}$. Under this assumption, we shall show that there is an automorphism $\sigma \in \operatorname{Aut}(G)$ which exchanges $y_{1}$ and $y_{3}$, fixing the edge $x_{2} y_{2}$; this implies the claim.

Consider a 3-coloring $c: V(G) \rightarrow\{1,2,3\}$ with $c\left(x_{1}\right)=c\left(x_{2}\right)=c\left(x_{3}\right)=3$. Since $G$ is not 3-distinguishing colorable, there is a non-trivial automorphism $\sigma \in \operatorname{Aut}(G, c)$. If $\sigma\left(y_{2}\right)=y_{2}$, then $\sigma$ exchanges $x_{1}$ and $x_{3}$, fixing $x_{2}$; otherwise, $\sigma$ would be the identity map. This implies that $\sigma$ exchanges $y_{1}$ and $y_{3}$, fixing $x_{2} y_{2}$ and hence it is the desired one in this case. Thus, we may assume that $\sigma\left(y_{2}\right)$ is another vertex different from $y_{2}$, say $z$, and is adjacent to all of $x_{1}, x_{2}$ and $x_{3}$. If $z$ coincides with neither $y_{1}$ nor $y_{3}$, then at least one of $x_{1} z x_{2}$ and $x_{2} z x_{3}$ forms a corner of a face since $\operatorname{deg} z=4$, and either $\left\{x_{1}, x_{2}\right\}$ or $\left\{x_{2}, x_{3}\right\}$ would become a 2 -cut of $G$, which is contrary to $G$ being 3 -connected. Thus, we may assume that $z=y_{3}$, up to symmetry, and there is an edge $x_{1} y_{3}$.

Since $x_{1}$ is adjacent to $y_{1}, y_{1}^{\prime}, y_{2}$ and $y_{3}$ and has degree $4, x_{1}$ is not adjacent to $y_{3}^{\prime}$. The edge $x_{1} y_{3}$ blocks the existence of an edge between $x_{2}$ and $y_{3}^{\prime}$. Thus, we can define another 3-coloring $c^{\prime}: V(G) \rightarrow\{1,2,3\}$ of $G$ by $c^{\prime}\left(x_{1}\right)=c^{\prime}\left(x_{2}\right)=c^{\prime}\left(y_{3}^{\prime}\right)=3$ and there is a non-trivial automorphism $\sigma^{\prime} \in \operatorname{Aut}\left(G, c^{\prime}\right)$ with its extension $h^{\prime}$ since $G$ is not 3 -distinguishing colorable. Clearly, we have $h^{\prime}\left(A_{1}\right)=A_{1}$ since $\sigma^{\prime}\left(\left\{x_{1}, x_{2}\right\}\right)=\left\{x_{1}, x_{2}\right\}$. If $\sigma^{\prime}$ exchanges $x_{1}$ and $x_{2}$, then there must be an edge $\sigma^{\prime}\left(x_{1}\right) \sigma^{\prime}\left(y_{3}\right)=x_{2} y_{1}^{\prime}$. However, its pre-image $x_{1} y_{3}$ blocks the existence of such an edge by the planarity. This implies that $\sigma^{\prime}$ fixes $x_{1}, y_{1}, x_{2}, y_{2}$ and $y_{3}^{\prime}$, which would force $\sigma^{\prime}$ to be the identity map, a contradiction. Therefore, this is not the case and only the previous case happens as we want.

Now assume that $\operatorname{deg} y_{2}=3$ and let $x_{1} y_{2} x_{3} y_{4}$ be the boundary cycle of the third face incident to $y_{2}$. If $\operatorname{deg} x_{2}=4$, then we can follow our arguments in the last three paragraphs, exchanging $x_{i}$ and $y_{i}$, to show the existence of $\sigma$ and hence $\operatorname{deg} y_{1}=\operatorname{deg} y_{3}$ and $\operatorname{deg} x_{1}=\operatorname{deg} x_{3}$. Thus, we may assume that $\operatorname{deg} x_{2}=3$ and let $y_{1} x_{2} y_{3} x_{4}$ be the boundary cycle of the third face incident to $x_{2}$.

Suppose that $\operatorname{deg} y_{1} \neq \operatorname{deg} y_{3}$. Without loss of generality, we may assume that $\operatorname{deg} y_{1}$ $=3$ and $\operatorname{deg} y_{3}=4$. Applying the first case to the two faces sharing $x_{2} y_{3}$, we conclude that 
$\operatorname{deg} x_{3}=\operatorname{deg} x_{4}$ and $\operatorname{deg} y_{1}=\operatorname{deg} y_{2}=3$. Consider a 3-coloring $c: V(G) \rightarrow\{1,2,3\}$ of $G$ with $c\left(y_{2}\right)=c\left(y_{3}\right)=3$. Since $G$ is not 3-distinguishing colorable, there is a nontrivial automorphism $\sigma \in \operatorname{Aut}(G, c)$. Since $\operatorname{deg} y_{2} \neq \operatorname{deg} y_{3}=4, \sigma$ fixes $y_{2}$ and $y_{3}$, and hence it exchanges $x_{2}$ and $x_{3}$; it would be the identity map otherwise. Thus, we have $\operatorname{deg} x_{2}=\operatorname{deg} x_{3}=\operatorname{deg} x_{4}=3$ and $\operatorname{deg} y_{1}=\operatorname{deg} y_{4}=3$.

If $\operatorname{deg} x_{1}=3$, then there must be an edge $x_{4} y_{4}$ so that $x_{1} y_{1} x_{4} y_{4}$ bounds a face and we find that $y_{3}$ would be a cut vertex of $G$, contrary to $G$ being 3 -connected. Thus, we have $\operatorname{deg} x_{1}=4$. Let $y_{5}$ be the fourth neighbor of $x_{1}$ and let $x_{5}$ be the fourth neighbor of $y_{3}$. The degree conditions we have concluded imply that $G$ consists of a cycle $y_{1} x_{2} y_{2} x_{3} y_{4} x_{5} y_{5} x_{4}$ of length 8 and the vertices $x_{1}$ and $y_{3}$ of degree $4 ; x_{1}$ is adjacent to all $y_{i}$ 's but $y_{3}$ while $y_{3}$ is adjacent to all $x_{i}$ 's but $x_{1}$. Consider a 3-coloring $c^{\prime}: V(G) \rightarrow\{1,2,3\}$ of $G$ with $c^{\prime}\left(y_{1}\right)=c^{\prime}\left(x_{3}\right)=3$. Since the cycle of length 8 contains all vertices of degree 3 in $G$, any automorphism $\sigma^{\prime} \in \operatorname{Aut}\left(G, c^{\prime}\right)$ preserves the cycle and fixes $y_{1}$ and $x_{3}$. This implies that $\sigma^{\prime}$ would be the identity map, contrary to $G$ not being 3 -distinguishing colorable. Therefore, we have $\operatorname{deg} y_{1}=\operatorname{deg} y_{3}$ and $\operatorname{deg} x_{1}=\operatorname{deg} x_{3}$ similarly.

Now we have just proved Claim 1. By the claim, we can conclude that the degrees appear around all faces in the same pattern. If there are adjacent vertices of degree 3 , then the claim forces all of their neighbors to have degree 3 and we find that $G$ is isomorphic to $Q_{3}$, which is one of the exceptions in the theorem. Therefore, there are only two patterns of degrees around a face, (i) $(3,4,3,4)$ and (ii) $(3,4,4,4) ;(4,4,4,4)$ is excluded since $G$ has eight vertices of degree 3 . However, it is clear that $G$ is isomorphic to $R\left(Q_{3}\right)$ in Case (i), which should be excluded as the second exception.

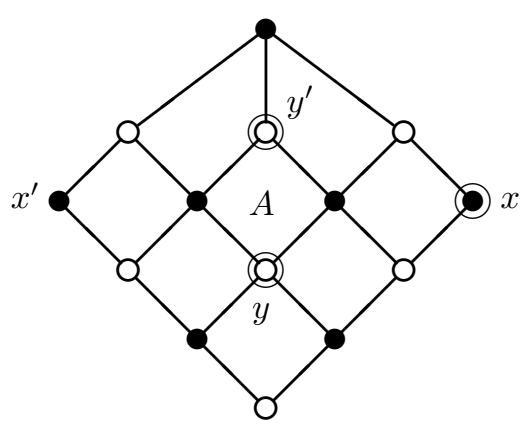

Figure 2: A local structure of $G$

On the other hand, we find that $G$ is isomorphic to $R\left(R\left(Q_{3}\right)\right)$, with the local structure depicted in Figure 2 in Case (ii), as follows. First look at a face $A$ with two white vertices $y$ and $y^{\prime}$ and suppose that $\operatorname{deg} y=4$ and $\operatorname{deg} y^{\prime}=3$. The four faces incident to $y$ form a big square containing $y$ inside. Its four corners are white vertices of degree 3 and its sides are divided by black vertices of degree 4 by Claim 1 . We can find such a square for each white vertex of degree 4 and conclude that such six squares form a cube including the whole of $G$. If we denote the cube by $Q_{3}$, then $G$ can be regarded as $R\left(R\left(Q_{3}\right)\right)$.

The local structure of $G$ in Figure 2 consists of fourteen distinct vertices and the black vertices have degree 4. Consider a 3-coloring $c$ of $G$ such that the two white vertices and the one black vertex encircled get color 3. Take any automorphism $\sigma \in \operatorname{Aut}(G, c)$ with its extension $h$. Since $\operatorname{deg} y \neq \operatorname{deg} y^{\prime}, \sigma$ fixes $y, y^{\prime}$ and $x$. Furthermore, $h$ cannot flip the face 
$A$ since $x \neq x^{\prime}$. This implies that $\sigma$ is the identity map. Therefore, $c$ is a 3 -distinguishing coloring and hence $G$ would be 3 -distinguishing colorable, contrary to our assumption on $G$. Thus, this is not the case and the theorem follows.

\section{For further studies}

Following our proof of Theorem 1.2, we can conclude that $\chi_{D}(G) \leq \chi(G)+\alpha$ for a small integer $\alpha=1$ or 2 in each case. More detailed arguments could give a kind of structural characterization on the distinguishing chromatic numbers of 3-connected planar graphs. The case of planar triangulations has been settled in [7] by Sano.

\section{References}

[1] M. O. Albertson and K. L. Collins, Symmetry breaking in graphs, Electronic J. Combin. 3 (1996), R18.

[2] B. Bogstad and L. J. Cowen, The distinguishing number of the hypercube, Discrete Math. 283 (2004), 29-35.

[3] K. L. Collins and A. Trenk, The distinguishing chromatic number, Electronic J. Combin. 13 (2006), R16.

[4] T. Fukuda, S. Negami and T. Tucker, 3-Connected planar graphs are 2-distinguishable with few exceptions, Yokohama Math. J. 54 (2008), 143-153.

[5] S. Negami, Uniqueness and faithfulness of embedding of toroidal graphs, Discrete Math. 44 (1983), 161-180.

[6] S. Negami and S. Sakurai, Distinguishing chromatic numbers of planar graphs, Yokohama Math. J. 55 (2010), 179-188.

[7] T. Sano, The distinguishing chromatic number of triangulations on the sphere, to appear in Yokohama Math. J., 2011.

[8] H. Whitney, Congruent graphs and the connectivity of graphs, Amer. J. Math. 54 (1932), 150168. 